This is the submitted version of the article:

Laguna-Marco, M.A.; Piquer, C.; Roca, A.G.; Boada, R.; Andrés-Vergés, M.; Veintemillas-Verdaguer, S.; Serna, C.J .; Iadecola, A.; Chaboy, J.. Structural determination of Bi-doped magnetite multifunctional nanoparticles for contrast imaging. Physical Chemistry Chemical Physics, (2014). 16. 34: 18301 - . 10.1039/c4cp01392a.

Available at: https://dx.doi.org/10.1039/c4cp01392a 


\title{
Structural determination of Bi-doped magnetite multifunctional nanoparticles for contrast imaging ${ }^{\dagger}$
}

\author{
M. A. Laguna-Marco, ${ }^{* a}$ C. Piquer, ${ }^{a}$ A. G. Roca, ${ }^{b}$ R. Boada, ${ }^{c}$ M. Andrés-Vergés, ${ }^{d}$ S. Veintemillas- \\ Verdaguer, ${ }^{e}$ C. J. Serna, ${ }^{e}$ A. Iadecola, ${ }^{f}$ and J. Chaboy ${ }^{a}$
}

Received Xth $X X X X X X X X X X 20 X X$, Accepted Xth $X X X X X X X X X 20 X X$

First published on the web $X$ th $X X X X X X X X X X 200 X$

DOI: 10.1039/b000000x

To determine with precision how $\mathrm{Bi}$ atoms are distributed in Bi-doped iron oxide nanoparticles their structural characterization has been carried out by X-ray absorption spectroscopy (XAS) recorded at the K edge of $\mathrm{Fe}$ and at the $\mathrm{L}_{3}$ edge of Bi. The inorganic nanoparticles are nominally hybrid structures integrating an iron oxide core and a bismuth oxide shell. The XAS spectra show that, in the samples synthesized by precipitation in aqueous media and laser pyrolysis, the $\mathrm{Bi}$ atoms neither enter in the iron oxide spinel lattice nor form any other mixed Bi-Fe oxide. No modification of the local structure around the Fe atoms induced by the $\mathrm{Bi}$ atoms is observed at the $\mathrm{Fe} \mathrm{K}$ edge. In addition, contrary to expectations, our results indicate that the $\mathrm{Bi}$ atoms do not form a well defined $\mathrm{Bi}$ oxide structure. The XAS study at the $\mathrm{Bi} \mathrm{L}_{3}$ edge indicates that the environment around $\mathrm{Bi}$ atoms is highly disordered and only a first oxygen coordination shell is observed. Isolated $\left[\mathrm{BiO}_{6-x}(\mathrm{OH})_{x}\right]$ units (or similar) bonded through hydroxyl bridges to the nanoparticle, rather than a well defined $\mathrm{Bi}_{2} \mathrm{O}_{3}$ shell, are surrounding the nanoparticle. On the other hand, the XAS study indicates that, in the samples synthesized by thermal decomposition, the Bi atoms are embedded in a longer-range ordered structure showing first and second neighbors.

\section{Introduction}

Multifunctional nanoparticles combining magnetic and optical properties in a single entity have been recently proposed as new-generation contrast and therapeutic agents in biomedicine 1,2 . To date, superparamagnetic iron oxide nanoparticles have been seen as the most promising materials for many medical applications due to their magnetic properties, chemical stability, biocompatibility and low price. ${ }^{3-5}$ Together with $\mathrm{Gd}$ chelates, superparamagnetic iron oxide nanoparticles are the most used contrast agents for the detection of tumors by Magnetic Resonance Imaging (MRI) ${ }^{3,6}$. However, in some cases, imaging results are too vague to provide detailed enough bio-

† Electronic Supplementary Information (ESI) available: [details of any supplementary information available should be included here]. See DOI: $10.1039 / \mathrm{b} 000000 \mathrm{x} /$

${ }^{a}$ Instituto de Ciencia de Materiales de Aragón and Departamento de Física de la Materia Condensada , CSIC - Universidad de Zaragoza, 50009 Zaragoza, Spain

${ }^{b}$ ICN2 - Institut Catala de Nanociencia i Nanotecnologia, Campus UAB, 08193 Bellaterra (Barcelona), Spain

${ }^{c}$ Diamond Light Source Ltd, Harwell Science and Innovation Campus, Chilton, Didcot, Oxfordshire, OX11 ODE, UK

${ }^{d}$ Departamento de Química Orgánica e Inorgánica, Universidad de Extremadura, 06071 Badajoz, Spain

e Instituto de Ciencia de Materiales de Madrid, CSIC, Cantoblanco 28049 Madrid, Spain

${ }^{f}$ Elettra-Sincrotrone Trieste S.C.p.A., s.s. 14-km 163.5, 34149 Basovizza, Trieste, Italy logical information ${ }^{7}$. To further improve the sensitivity and efficiency in imaging techniques many efforts focus nowadays on synthesizing hybrid nanostructures where each of the components of the heterostructure is aimed at an specific task. Thus, magneto-luminiscent nanoparticles have been proposed for diagnosis imaging, but this class of materials present serious drawbacks related with the luminescent properties ${ }^{8}$. Alternatively, multifunctional particles combining good magnetic response and high X-ray absorption coefficient, for instance including a heavy element such as bismuth, emerge as a promising tool in the field ${ }^{9}$. These particles are able to be imaged by both MRI and Computed Tomography (CT) providing higher sensitivity. This approach, however, has not been explored much yet, even when $\mathrm{Bi}$ has a long bioutility history and $\mathrm{CT}$ is one of the most common imaging techniques.

Recent works on Bi-doped nanoparticles have suggested that the Bi atoms do not enter the spinel lattice, but form a core/shell magnetite/bismuth oxide heterostructure ${ }^{14}$. According to these results, Bi acts as surfactant controlling particle size and colloidal properties. Although the formation of a bismuth oxide phase was proposed, no detailed structural study of this Bi phase has been performed yet in this type of nanoparticles. The location and type of bonds of Bi determine to a high extent the size, shape and physicochemical properties (stability or degradation in biological media, partial release or toxicity) of the nanoparticles and so their functionalization and application as double contrast agents for imaging. Therefore 
an accurate determination of the environment around $\mathrm{Bi}$ is of paramount importance.

To provide a microstructural determination of how the bismuth atoms enter these systems we present here a detailed X-ray Absorption Spectroscopy (XAS) study on several Bidoped iron oxide nanoparticles with sizes below the monodomain diameter (6-30 $\mathrm{nm}$ range), different amount of $\mathrm{Bi}$ doping and synthesized by up to three different routes. Both energy regions of the XAS spectra, i.e. X-ray Absorption Near Edge Structure (XANES) and Extended X-ray Absorption Fine Structure (EXAFS), have been recorded at the $\mathrm{K}$ edge of iron and and $\mathrm{L}_{3}$ edge of bismuth. The analysis of the modulations in the X-ray absorption coefficient recorded at both regions provide complementary information about the local structure around the absorbing atoms: distances to, number and nature of neighboring atoms as well as type of coordination (geometry) around the absorbing atoms. XAS is an atomic specific experimental probe that does not require any long range crystal symmetry and hence permits us to have direct access to the local atomic structure of a wide variety of materials, including amorphous materials, liquids and nanoparticles. The study of the XAS recorded at the Fe K edge will let us know the environment around $\mathrm{Fe}$ atoms and whether the nanoparticles fully correspond to the nominal $\mathrm{Fe}_{3} \mathrm{O}_{4}$ magnetite or not. The $\mathrm{Bi}_{3}$-edge absorption spectrum will provide the accurate determination of the local environment around $\mathrm{Bi}$.

For the analysis of the signals recorded at the Fe edge, we have compared both XANES and EXAFS signals to a number of references. In addition, by using the Fourier transform of the EXAFS signal (FT, which roughly speaking gives us the atomic radial distribution function in R-space) the evolution of the environment around Fe with the amount of Bi doping has been studied. To this aim, the experimental data have been compared to theoretical FEFF calculations. Regarding the spectra recorded at the $\mathrm{Bi} \mathrm{L}_{3}$ edge, as a first step we have compared the signals of the nanoparticles to a number of reference compounds. Further analysis of the atomic distribution around the $\mathrm{Bi}$ atom has been carried out by comparing the experimental signals (including Fourier transforms and Fourier back-transforms i.e. Fourier filtered n-shell EXAFS oscillations), to $a b$-initio computations.

\section{Experimental and computational methods}

Fe oxide nanoparticles containing different amounts of bismuth were synthesized following three different routes: laser pyrolysis (LP), precipitation in aqueous media (PAM) and thermal decomposition (TD). In all the synthesis we tried to dope iron oxide nanoparticles with less than $10 \%$ in bismuth to reach similar sizes than the ones published before ${ }^{14,15}$

Samples PAM1, PAM2 and PAM7 were synthesized by precipitating iron(II) and bismuth(III) nitrate in a mixture of al- kaline media and ethanol. The reaction was carried out in a three-necked $500 \mathrm{~cm}^{3}$ round-bottom flask placed in an oil bath with mechanical stirring using a dual glass blade and under nitrogen flow. In a typical experiment, a mixture of iron and bismuth salts (different molar Fe/Bi ratios) in $20 \mathrm{~mL}$ of water $0.02 \mathrm{M} \mathrm{H}_{2} \mathrm{SO}_{4}, 0.07 \mathrm{M} \mathrm{NaOH}$ and $0.1 \mathrm{M} \mathrm{KNO}_{3}(180$ $\mathrm{mL}$ water/ethanol 80:100), were degasified for $1 \mathrm{~h}$ by nitrogen bubbling and mixed at room temperature. When the precipitation was completed, the system was heated to $90^{\circ} \mathrm{C}$ and left undisturbed for $24 \mathrm{~h}$. Then the suspension was cooled at room temperature with an ice bath, and the solid was separated by magnetic decantation and washed several times with distilled water. $^{15}$

Sample LP10 was obtained by the decomposition of iron pentacarbonyl $\left(\mathrm{Fe}(\mathrm{CO})_{5}, 30{ }^{\circ} \mathrm{C}\right)$ and bismuth triethyl (TBI, $7.5^{\circ} \mathrm{C}$ ) after heating a mixture of argon and air with ethylene under the action of a continuous-wave carbon dioxide laser $\left(\lambda=10.60 \pm 0.05 \mu \mathrm{m}\right.$, energy density $\left.=652 \mathrm{Wcm}^{-2}\right) .{ }^{16,17}$ Above a certain pressure and laser power, a critical concentration of product nuclei is formed in the reaction zone. This leads to homogeneous nucleation of particles that are carried to a filter by an inert gas. Other constant parameters of the experiment were: laser power, $82 \mathrm{~W}$; argon axial flux, $15 \mathrm{sccm}$; argon windows flux, $220 \mathrm{sccm}$; pressure, 400 mbar; reactive temperature, $20{ }^{\circ} \mathrm{C}$; TBI carrier gas was $\mathrm{N}_{2} 4 \mathrm{sccm}$; $\mathrm{Fe}(\mathrm{CO})_{5}$ carrier gas was a mixture of air $3.1 \mathrm{sccm}$ and ethylene $7 \mathrm{sccm}$; average duration of the experiment, $5 \mathrm{~h}$.

Sample TD03 consists on bismuth doped nanoparticles obtained by thermal decomposition of $\mathrm{Fe}(\mathrm{acac})_{3}$ and $\mathrm{Bi}$ (acet $)_{3}$ in the presence of oleic acid and oleylamine as surfactants, and benzyl ether. ${ }^{18}$ A mixture of $5.03 \mathrm{~g} \mathrm{Fe}(\mathrm{acac})_{3}(14.25 \mathrm{mmol})$, $0.290 \mathrm{~g} \mathrm{Bi}(\text { acet })_{3}(0.75 \mathrm{mmol}), 16.93 \mathrm{~g} \mathrm{1,2-dodecanediol} \mathrm{(75}$ $\mathrm{mmol}), 14.19 \mathrm{~g}$ oleic acid (45 mmol), $17.27 \mathrm{~g}$ oleylamine (45 $\mathrm{mmol}$ ), and $150 \mathrm{~mL}$ benzyl ether was put into a four-necked flask and heated up to $200{ }^{\circ} \mathrm{C}$ for 120 min with magnetically stirring and under a nitrogen flow. Then, the solution was heated to reflux (b.p. $\sim 300{ }^{\circ} \mathrm{C}$ ) for $30 \mathrm{~min}$ in a $\mathrm{N}_{2}$ atmosphere. To remove impurities, the solution at room temperature was mixed with ethanol, centrifuged at $5650 \mathrm{~g}$ for $10 \mathrm{~min}$ and the supernatant discarded. Finally, nanoparticles were mixed with $40 \mathrm{ml}$ of hexane and $0.1 \mathrm{ml}$ of oleic acid, and centrifuged twice at $5650 \mathrm{~g}$ for $10 \mathrm{~min}$. Powders from these suspensions were obtained by precipitation with ethanol and dryness at room temperature under a $\mathrm{N}_{2}$ flow.

Morphology, particle size, and distribution were investigated using a transmission electron microscope (JEOL-2000 FXII) operating at $200 \mathrm{keV}$. HRTEM images were obtained by means of a Philips TECNAI 200 T. Mean size (D) and the standard deviation (SD) associated with it were evaluated from the electron micrographs by counting around 500 particles. The microstructure of the obtained nanoparticles was analyzed by powder X-ray diffraction (XRD) at room temperature, using 
a rotating-anode Rigaku diffractometer in the Bragg-Brentano geometry, with $\mathrm{Cu}-\mathrm{K} \alpha$ radiation. The crystallite size was determined from the full-width at half-maximum of the reflection (311) of magnetite by using the Scherrer equation. Iron and bismuth content in the magnetite samples was determined by plasma emission (ICP, PERKIN ELMER OPTIMA 2100 $\mathrm{DV})$. For this analysis, $25 \mathrm{mg}$ of powder were first dissolved with concentrated $\mathrm{HCl}$ and then diluted with doubly distilled water.

Fe K-edge and $\mathrm{Bi}_{3}$-edge XAS spectra were recorded at the bending magnet XAFS beamline of the ELETTRA Synchrotron Facility (Trieste, Italy) ${ }^{19}$. Experiments were performed at room temperature and in the transmission mode using three ionization chambers mounted in series for simultaneous measurements on the sample and a reference. Fe and $\mathrm{Bi}$ foils were used as reference for calibration checking purposes. Measurements were also performed in the fluorescence mode by using a single-element Si-drift detector (SDD). No difference, other than a lower signal to noise ratio, was found with respect to the transmission ones. The energy of the incoming X-ray beam was tuned with a $\mathrm{Si}(111)$ double-crystal monochromator to probe the absorption at the Fe K edge and the $\mathrm{Bi} \mathrm{L}_{3}$ edge. Harmonic rejection was achieved by detuning the second crystal from parallel alignment at Fe K-edge energy.

Standard $\sim 13 \mathrm{~mm}$-diameter pellets of the powdered samples thoroughly mixed with boron nitride were made. Total thickness of the samples was in the $300-700 \mu \mathrm{m}$ range to assure they could be handled without breaking. The amount of sample was optimized to obtain the best signal-to-noise ratio ( $~ 0.7$ edge jump height at the Fe K edge).

A number of reference samples have also been measured: bulk $\mathrm{Fe}_{3} \mathrm{O}_{4}$ (magnetite), $\quad \gamma-\mathrm{Fe}_{2} \mathrm{O}_{3}$ (maghemite), $\alpha-\mathrm{Fe}_{2} \mathrm{O}_{3}$ (hematite), $\mathrm{FeO}(\mathrm{OH})$ (goethite) and $\mathrm{FeO}$ (wustite) at the $\mathrm{Fe}$ $\mathrm{K}$ edge and $\mathrm{Bi}$ metal, $\mathrm{BiFeO}, \alpha-\mathrm{Bi}_{2} \mathrm{O}_{3}, \beta-\mathrm{Bi}_{2} \mathrm{O}_{3}, \mathrm{Bi}\left(\mathrm{OH}_{3}\right)$ and the precursors $\mathrm{Bi}(\text { acet })_{2}$ and $\mathrm{Bi}\left(\mathrm{NO}_{3}\right)_{3}$ at the $\mathrm{Bi}_{3}$ edge.

The EXAFS spectra were analyzed according to standard procedures ${ }^{20}$ using the HORAE-IFEFFIT (Athena, Artemis) program package and the FEFF code for the ab-initio multiple scattering EXAFS simulations. ${ }^{21,22}$ The ab-initio computation of the XANES spectra was carried out using the multiplescattering code Continuum ${ }^{23}$ included in the MXAN package $^{24}$. For the computations the potential for the different atomic clusters was approximated by a set of spherically averaged muffin-tin (MT) potentials built by following the standard Mattheis' prescription. The muffin-tin radii were determined following the Norman's criterion. For the reference compounds we have first determined the minimum size of the cluster needed to reproduce all the features of the experimental XANES spectrum. This is made by adding progressively coordination shells surrounding the absorbing atom until convergence is obtained, i.e. the addition of further shells do not lead to the modification of the relative energy separation and intensity of the spectral features or to the appearance of new ones. Then, care was taken to determine the best choice for the overlapping factor (ovf) between the muffin-tin spheres and for the exchange and correlation components of the final state potential. ${ }^{25-27}$ Thus, in a second step we have checked the influence of the ovf used to built up the scattering potential into the reproduction of the experimental XANES spectra. Moreover, we have also studied the effect of modifying the value of the maximum angular momentum quantum number, $l_{\text {max }}$, needed to account for the experimental absorption spectrum in the first $\sim 50 \mathrm{eVs}$. Last but not least we have studied the best choice of the exchange and correlation part of the final state potential (ECP) to reproduce not only the spectral shape but also the intensity of the computed spectral features and their relative energy separation. To this end we have performed the computations by using (i) the energy dependent Hedin-Lundqvist (HL) ECP potential, (ii) the energy dependent Dirac-Hara (DH) ECP potential, (iii) only the real part of the HL ECP (hereafter real HL) and (iv) by adding the imaginary part of the HL ECP to the Dirac-Hara one (hereafter complex Dirac-Hara). The best reproduction of the experimental XANES spectra was obtained by using the Dirac-Hara ECP potential and by imposing a $1 \%$ of overlapping factor, in agreement to previous works for transition metal oxides and related compounds ${ }^{27-29}$. In order to get an enhanced view of the differences the results obtained in the case of using the real DH ECP are shown. A complete discussion of the procedure can be found elsewhere ${ }^{30-32}$. The theoretically calculated spectra have been directly compared to the experimental XANES spectrum i.e., no fitting procedure (free parameter) has been used during the calculations. The assessment of the quality of the theoretical computations is based on the correct reproduction of the shape and energy position of the different spectral features and of their relative energy separation and the intensity ratio. For the rest of the computations we have just determined the minimum size of the cluster needed to reproduce all the features of the experimental XANES spectrum. The overlapping factor and the exchange and correlation components of the final state potential have been fixed to those found for the reference compound. In all the cases, the theoretical spectra have been convoluted with a Lorentzian shape function ( $\Gamma=1.5 \mathrm{eV}$ for $\mathrm{Fe} \mathrm{K}$ edge, $5.98 \mathrm{eV}$ for $\mathrm{Bi}_{3}$ edge) to account for the core-hole lifetime ${ }^{33}$ and the experimental resolution.

\section{Results and discussion}

\subsection{TEM and XRD Structural Characterization}

The studied samples, synthesized by the different methods described above, are listed in table 1. The Bi content, parti- 


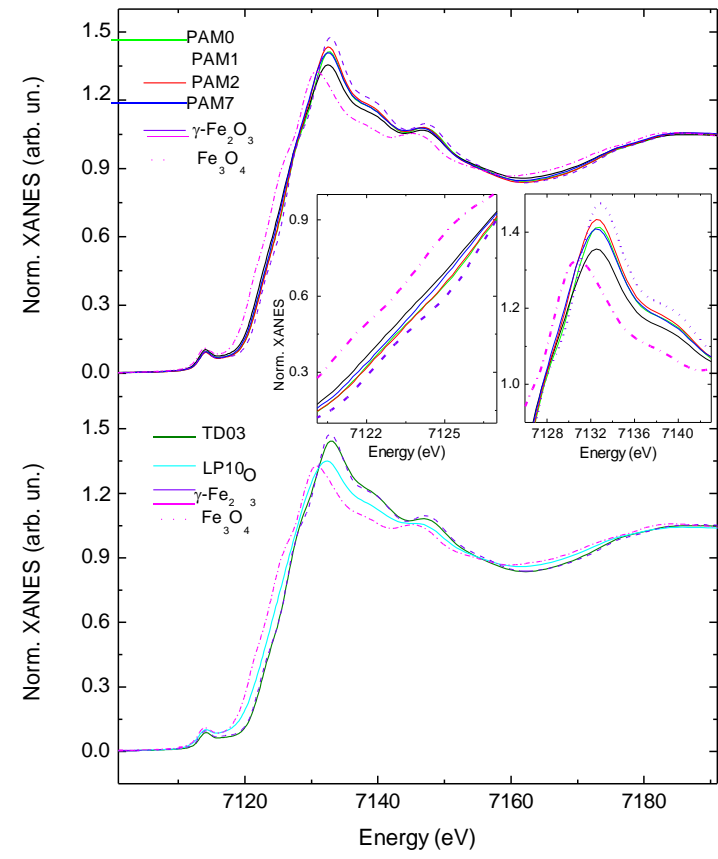

Fig. 1 (Experimental Fe K-edge XANES spectra of bismuth-doped iron oxide nanoparticles and reference samples. Insets: zoomed threshold (left) and white line (right) regions .

cle size measured by TEM and crystallite size calculated from Scherrer's equation $\left(\mathrm{D}_{X R D}\right)$ are also summarized in the table.

Representative TEM images of the nanoparticles synthesized by the three methods and the X-ray diffractograms of all the samples are reported in the supplementary information. XRD patterns of all the samples show a main phase that corresponds to iron oxide with an inverse spinel structure, either magnetite or maghemite.

\subsection{Fe K-edge XANES and EXAFS Characterization}

The Fe K-edge XANES spectra of all the measured Bi-doped iron oxide nanoparticles are shown in Fig. 1. The spectra corresponding to bulk $\mathrm{Fe}_{3} \mathrm{O}_{4}$ (magnetite) and $\gamma$ - $\mathrm{Fe}_{2} \mathrm{O}_{3}$ (maghemite) references are also included. All the Bi-doped samples present an absorption profile that lies in between those of maghemite and magnetite references. The presence of precursors as well as the formation of other Fe oxides or hydroxides can be discarded by simple comparison of the spectral shapes (see supplementary information). Further confirmation has been obtained by fitting the spectra of the nanoparticles to the weighted addition of reference spectra following the procedure previously used by Piquer et. al. ${ }^{34}$ This procedure gives no appreciable amount of these references for the best fits (not shown).
No crystallographic change due to the $\mathrm{Bi}$ atoms entering in the iron-oxide nanoparticles can be observed. It should be noted that in the case of the native oxides the average $\mathrm{Fe}-\mathrm{O}$ interatomic distance is $\sim 1.98 \AA$ while the nominal average $\mathrm{Bi}-\mathrm{O}$ distance is $\sim 2.50 \AA$ for both $\alpha$ - and $\beta-\mathrm{Bi}_{2} \mathrm{O}_{3}$. Consequently, if $\mathrm{Bi}$ entered the iron oxide by substituting $\mathrm{Fe}$, there would be an structural distortion, through the modification of the $\mathrm{Fe}(\mathrm{Bi})-\mathrm{O}$ and $\mathrm{Fe}(\mathrm{Bi})-\mathrm{O}-\mathrm{Fe}(\mathrm{Bi})$ interatomic distances, that should be reflected in the absorption spectra. In the case of XANES the modification of these distances should imply both the marked shift of the edge position as well as changes in the overall spectral shape . None of these modifications has been detected. Similarly, changes are expected in the EXAFS part of the spectra if $\mathrm{Bi}$ substitutes Fe, but, as in the XANES case, these effects have not been observed. In particular, comparing the EXAFS spectra of the PAM series (see top panel of Fig. 2) no shift is observed in any of the spectral features, indicating no changes in the cell parameters and/or interatomic distances. Similarly, the amplitude of the EXAFS oscillations does not show any gradual evolution as the Bi content is increased. The lack of a Bi effect on the Fe K-edge spectra can be clearly seen when comparing the FT of the EXAFS signal corresponding to the experimental data and the FEFF simulations (middle and bottom panels of Fig. 2, respectively). According to the FEFF simulations, the Bi substitution should give rise to a gradual intensity decrease of both first and second peak as the Fe atoms are replaced by $\mathrm{Bi}$ in a 0,2 or $7 \%$. (See the supplementary information for the details about the EXAFS analysis and theoretical calculations.) The experimental data, however, show a random evolution with Bi content. Therefore, the differences observed between the spectra of the PAM series cannot be explained in terms of the presence of substitutional $\mathrm{Bi}$ in the iron oxide matrix. In the same way, if the bismuth atoms entered interstitially in the lattice a strong structural distortion and a concomitant gradual change in the profile of the XAS (both XANES and EXAFS) spectra should be expected, contrary to the observed behavior. On the other hand, the observed differences can be related to the formation of non-stoichiometric Fe oxides $\left(\mathrm{Fe}_{3-\delta} \mathrm{O}_{4}\right)$ due to the size reduction. The size reduction makes it very difficult to obtain nanoparticles that are perfect magnetite or maghemite, i.e. with exactly the same structural parameters and oxidation state as in the bulk compound. Indeed, differences like these ones are typically observed in iron oxide nanoparticles with no doping included. ${ }^{34}$ The crystallographic particularities of each non-stoichiometric oxide (i.e. number and type of vacancies, oxidation states, exact values of the lattice parameters, etc) depend on the details of the growth process undergone in each sample and will affect the profile of the XANES and EXAFS (and their FT). ${ }^{34}$

No modification of the local structure around the Fe atoms induced by the $\mathrm{Bi}$ atoms is observed at the Fe K edge. Truly, 
Table 1 Studied samples, preparation route, bismuth concentration determined by plasma emission (given in $\mathrm{Bi} / \mathrm{Fe}$ atomic ratio, $(\mathrm{Bi} / \mathrm{Fe})_{a t} \times$ $100)$, particle size measured by TEM ( $\mathrm{nm})$ and crystallite size calculated from XRD data $(\mathrm{nm})$.

\begin{tabular}{llccc}
\hline Sample & Preparation method & Bi \% & TEM size (nm) & XRD size (nm) \\
\hline PAM1 & Precipitation in aqueous media & 1 & $30 \pm 5$ & $27 \pm 1$ \\
PAM2 & Precipitation in aqueous media & 2 & $27 \pm 5$ & $21 \pm 1$ \\
PAM7 & Precipitation in aqueous media & 7 & $20 \pm 4$ & $13 \pm 1$ \\
\hline LP10 & Laser pyrolysis & 10 & $12 \pm 4$ & $5 \pm 1$ \\
\hline TD03 & Thermal decomposition & 0.3 & $6 \pm 2$ & $6 \pm 1$ \\
\hline
\end{tabular}
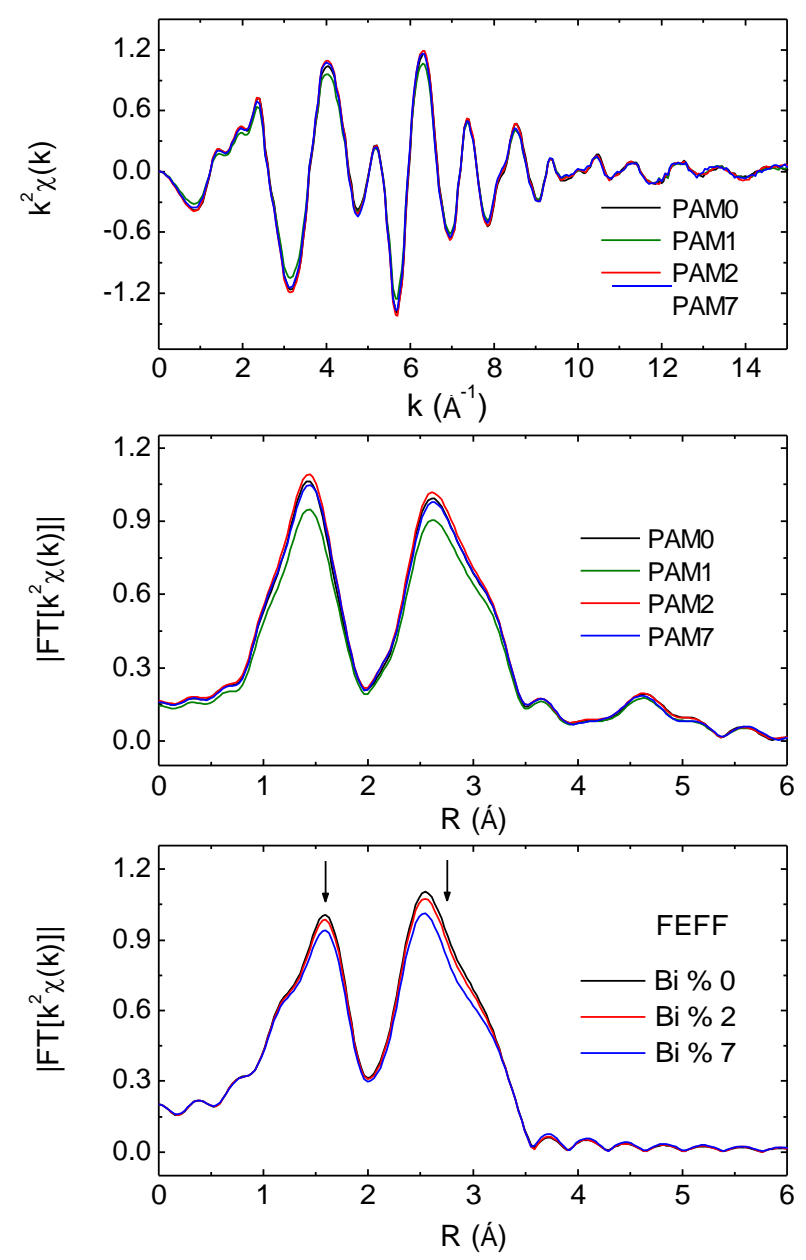

Fig. 2 (top) Comparison of the experimental Fe K-edge EXAFS spectra of the nanoparticles synthesized by precipitation in aqueous media with different Bi content: 0,1,2 and $7 \%$. (middle) modulus of the Fourier transform obtained from the experimental EXAFS signals. (bottom) FEFF calculated modulus of the Fourier transform for different $\mathrm{Bi}$ content: 0, 2 and 7\% substitutional $\mathrm{Bi}$ in the $\mathrm{Fe}_{3} \mathrm{O}_{4}$ crystal structure. an scenario where the effects of bismuth on the Fe signal are hindered in the averaged response of all the Fe atoms cannot be ruled out. In addition, it cannot be completely discarded that only a smaller part of the $\mathrm{Bi}$ atoms are located inside the nanoparticle. Nevertheless, the comparison in Fig. 2 suggests that the $\mathrm{Bi}$ atoms neither enter in the spinel structure substituting Fe nor in interstitial sites. Moreover, the structural environment of the Fe atoms remain unaltered and highly ordered. These conclusions are sustained on the analysis of both XANES and EXAFS regions of the spectra coupled with theoretical simulations. Since the bismuth atoms, are not located within the iron oxide lattice, they are expected to be in another phase around the iron oxide grain.

\subsection{Bi $\mathrm{L}_{3}$-edge XANES - PAM and LP samples}

The $\mathrm{Bi}_{3}$-edge XANES spectra of the samples are shown in Fig. 3 (a). As in the Fe $\mathrm{K}$ edge case, all the spectra corresponding to the samples under study are very similar (with the exception of TD03, which deserves special attention). A simple fingerprint analysis discards the presence of Bi-containing precursors, as well as $\mathrm{BiFeO}_{3}$ and metallic $\mathrm{Bi}$ (shown in the supporting information), as it can be expected from the synthesis reaction conditions and further purification processes made afterwards.

At first glance the spectra of our samples resemble that of $\alpha-\mathrm{Bi}_{2} \mathrm{O}_{3}$ and $\beta-\mathrm{Bi}_{2} \mathrm{O}_{3}$, see Fig. 3 (b). However, a closer inspection reveals noticeable differences. The spectral shape of our samples basically consists on a main absorption peak (A) located at $\sim 13452 \mathrm{eV}$, a slight shoulder-like structure just below the main peak (A2, $13440 \mathrm{eV})$ and a single oscillation after the main peak. This directly suggests that the local order around $\mathrm{Bi}$ is short-range like that found in amorphous materials ${ }^{20}$ and in metal ions in aqueous solution ${ }^{29,38,39}$. The spectral profile of both bismuth oxides, on the other hand, is richer. As can be seen in Fig. 3 (b) the spectra of $\alpha-\mathrm{Bi}_{2} \mathrm{O}_{3}$ and $\beta-\mathrm{Bi}_{2} \mathrm{O}_{3}$ present a more evident shoulder-like structure just below the edge ( $\mathrm{A} 1, \sim 13428 \mathrm{eV}$ ). In addition, the $\beta-\mathrm{Bi}_{2} \mathrm{O}_{3}$ compound shows a hump at $\mathrm{E} \sim 13465 \mathrm{eV}$ (B) followed by 


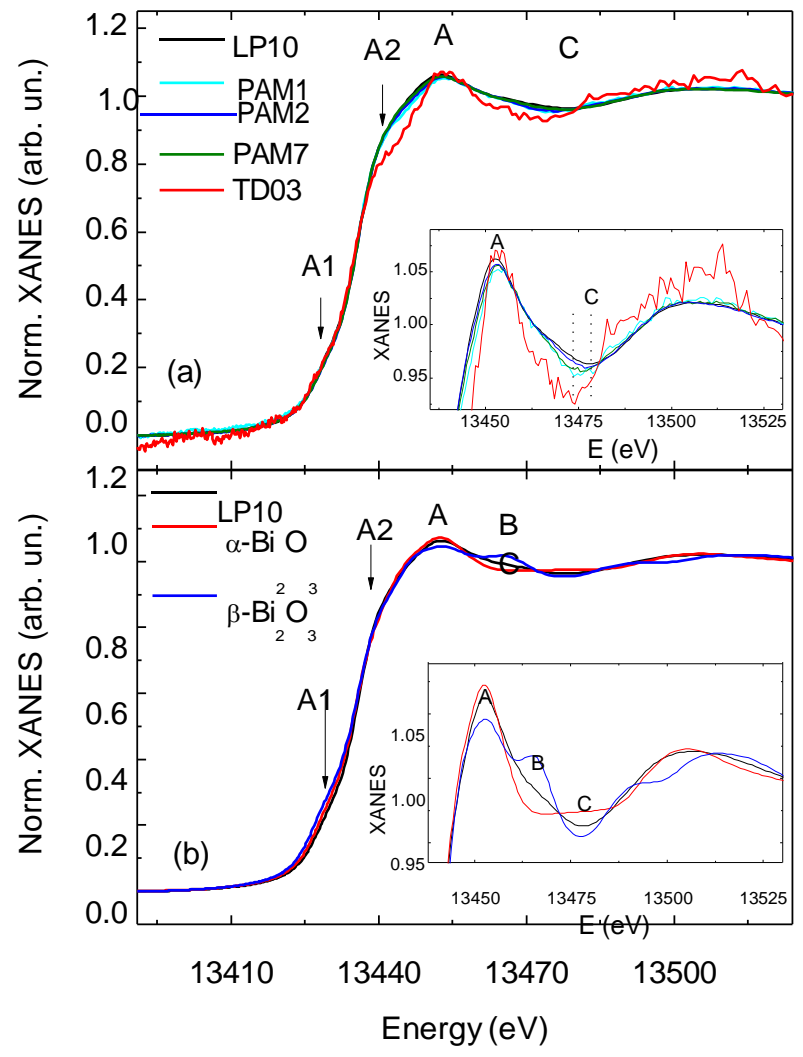

Fig. 3 (a) Comparison of the experimental $\mathrm{Bi}_{3}$-edge XANES spectra of all the bismuth-doped iron oxide nanoparticles. (b) Comparison of the experimental $\mathrm{Bi} \mathrm{L}_{3}$-edge XANES spectra of the

LP10 sample and the $\alpha-\mathrm{Bi}_{2} \mathrm{O}_{3}$ and $\beta-\mathrm{Bi}_{2} \mathrm{O}_{3}$ references.

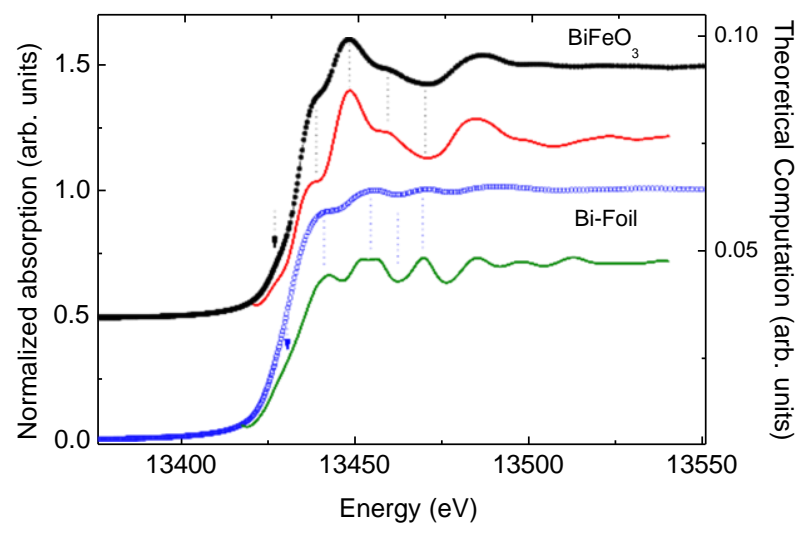

Fig. 4 Comparison between the experimental $\mathrm{Bi}_{3}$-edge XANES of metallic $\mathrm{Bi}$ (blue, $\circ$ ) and $\mathrm{BiFeO}_{3}$ (black, • ) and the computations performed by using the DH ECP and ovf $=1 \%$.

a negative feature at higher energies $(\mathrm{C})$ and the spectrum of $\alpha-\mathrm{Bi}_{2} \mathrm{O}_{3}$ presents a wide plateau at the energy region right after $\mathrm{A}$. Therefore, a more detailed study is needed to determine with higher precision if the local environment of $\mathrm{Bi}$ in the samples under study is just a distorted $\alpha-\mathrm{Bi}_{2} \mathrm{O}_{3}$ or $\beta-\mathrm{Bi}_{2} \mathrm{O}_{3}$, a mixture of both oxides or a different Bi environment. To this aim we have performed an study including theoretical compu-

tations.

To compare the XANES experimental spectra to the results of ab-initio $\mathrm{Bi} \mathrm{L}_{3}$-edge computations, we need first to find the more appropriate choice of several computational parameters by using well known reference compounds ${ }^{30,31}$. Thus, initially we have computed the theoretical spectrum for metallic $\mathrm{Bi}$ and $\mathrm{BiFeO}_{3}$. The results of the computations are reported in Fig. 4. The excellent agreement obtained allows us to fix several computational parameters as discussed in the experimental section. Hereafter all the calculations have been performed by using a $1 \%$ overlapping factor and the DH ECP potential.

Next, we have computed the XANES spectra for $\alpha-\mathrm{Bi}_{2} \mathrm{O}_{3}$ and $\beta-\mathrm{Bi}_{2} \mathrm{O}_{3}$ polymorphs ${ }^{40}$. Computations have been done for dif ferent cluster

ordination polyhedron, corresponding to the photoabsorbing $\mathrm{Bi}$ and its first oxygen coordination shell, and adding progressively further coordination shells. The results of these calculations, shown in Fig. 5, indicate that a good reproduction of the experimental spectra requires a cluster including, at least, two coordination shells. In this way the relative energy separation of the spectral features is accounted for by the calculations, especially regarding the high energy resonances $\mathrm{C}$ (in $\alpha-\mathrm{Bi}_{2} \mathrm{O}_{3}$ ) and $\mathrm{D}\left(\beta-\mathrm{Bi}_{2} \mathrm{O}_{3}\right)$. In other words, the inclusion of $\mathrm{Bi}-\mathrm{Bi}$ coordination is mandatory to account for the experimental spectra.

These computations are also compared to the spectra of the 
samples under study. As discussed above, the region at $\sim$ 

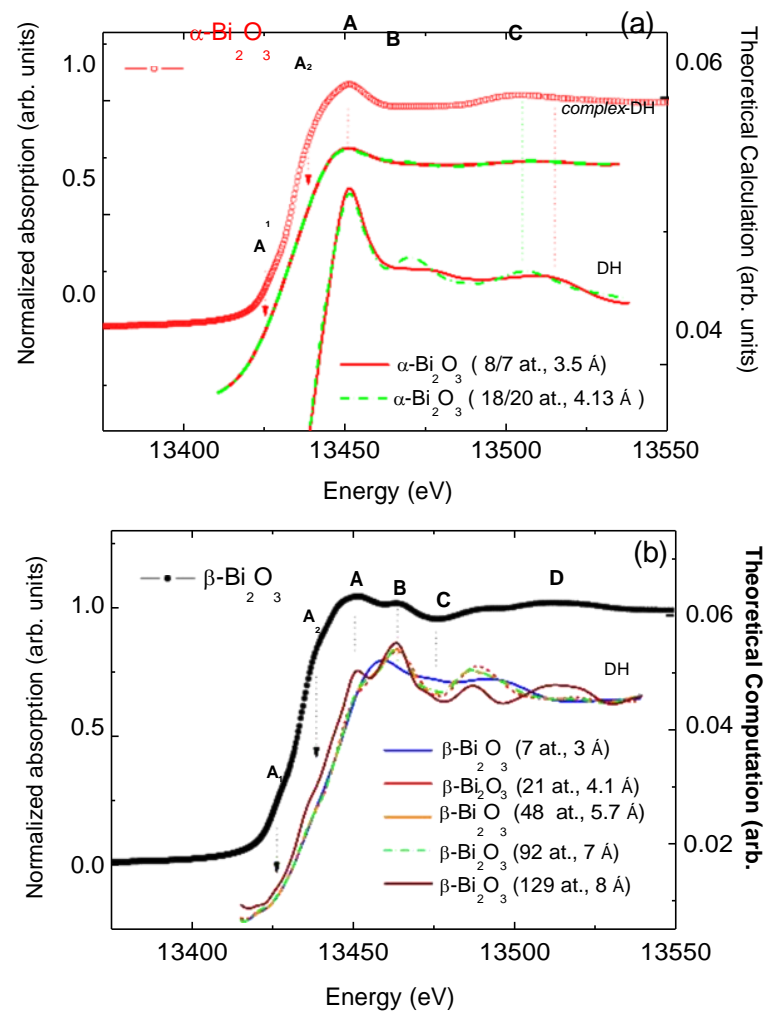

Fig. 5 (a) Comparison between the experimental $\mathrm{Bi}_{3}$-edge XANES of $\alpha-\mathrm{Bi}_{2} \mathrm{O}_{3}($ red, $\circ)$ and the theoretical calculations performed by usmg the complex DH ECP and by progressively increasing the cluster size (see text for details). In order to get an enhanced view of the differences the result of the computations obtained by using the real DH are also shown. (b) Same as above, using only the real DH computations, in the case of $\beta-\mathrm{Bi}_{2} \mathrm{O}_{3}$ (black, $\cdot$ ).
$13465 \mathrm{eV}$ in the XANES spectra of our samples cannot be accounted for in terms of the presence of $\alpha-\mathrm{Bi}_{2} \mathrm{O}_{3}$ or $\beta-\mathrm{Bi}_{2} \mathrm{O}_{3}$. To explain the XANES spectra of our samples one could suggest, in principle, that they contain a fraction of $\mathrm{Bi}$ atoms in a local arrangement similar to that of $\alpha-\mathrm{Bi}_{2} \mathrm{O}_{3}$ and also a fraction of $\mathrm{Bi}$ atoms in a $\beta-\mathrm{Bi}_{2} \mathrm{O}_{3}$ fashion. If this was the case, the theoretical computations above would indicate that the local order around $\mathrm{Bi}$ includes not only oxygen but also the second shell, implying the existence of Bi-O-Bi coordination. However, as we will see below, no Bi-O-Bi coordination can be detected in the nanoparticles synthesized by laser pyrolysis or precipitation in aqueous media.

Finally, we have also computed the XANES spectra by considering that $\mathrm{Bi}$ substitutes $\mathrm{Fe}$ in several iron oxides: magnetite, maghemite and hematite. These theoretical results do not agree with the experimental spectra (see the Supporting Information). This indicates that $\mathrm{Bi}$ does not enter in the spinel structure, as suggested by the results at the Fe K edge.

\subsection{Bi $\mathrm{L}_{3}$-edge EXAFS - PAM and LP samples}

The initial inspection of the EXAFS spectra verifies the absence of precursors, $\mathrm{BiFeO}_{3}$ and segregated metallic $\mathrm{Bi}$ (see supplementary information). It also points out that the EXAFS spectral shape of our samples resembles to some extent that of $\alpha-\mathrm{Bi}_{2} \mathrm{O}_{3}$ and $\beta-\mathrm{Bi}_{2} \mathrm{O}_{3}$, but it is notably less structured than that of either $\alpha-\mathrm{Bi}_{2} \mathrm{O}_{3}$ or $\beta-\mathrm{Bi}_{2} \mathrm{O}_{3}$ (see Fig. 6). The profile of our samples consists of basically only one oscillation, whereas the $\mathrm{Bi}_{2} \mathrm{O}_{3}$ references show extra features reflecting the existence of additional scattering paths with significant contribution, i.e. longer range order. Therefore, the simpler profile of our samples can be addressed to the short-range character of the structural order around Bi. Fig. 6 also shows the EXAFS spectrum of a commercial $\mathrm{Bi}(\mathrm{OH})_{3}$ reference. In the case of $\mathrm{Bi}(\mathrm{OH})_{3}$ the simple profile of its EXAFS indicates short-range character of the structural order around $\mathrm{Bi}$, similarly to LP10 and PAM7. It is worth to mention at this point that no detailed structural information has been reported for $\mathrm{Bi}(\mathrm{OH})_{3}$, which is not a stable and well-defined compound. The lack of detailed crystallographic information (space group, lattice parameters, atomic positions, etc) prevents from performing any precise analysis of its EXAFS signal.

The short-range character of the structural order around $\mathrm{Bi}$ in the LP and PAM samples is also inferred from the comparison of the Fourier transforms (FT). As it can be seen in the right panel of Fig. 6 all the compounds show a main peak at $\sim 1.6 \AA$ corresponding to a first coordination shell made of oxygen atoms, but our samples have only a very small bump at $\sim 3.2 \AA$, whereas the $\mathrm{Bi}_{2} \mathrm{O}_{3}$ references show a double peak due to Bi neighbors.

The analysis of the first peak of the FT shows that in the case of $\beta-\mathrm{Bi}_{2} \mathrm{O}_{3}$ the $\mathrm{Bi}-\mathrm{O}$ bond distance is smaller $(\sim 0.06$ 


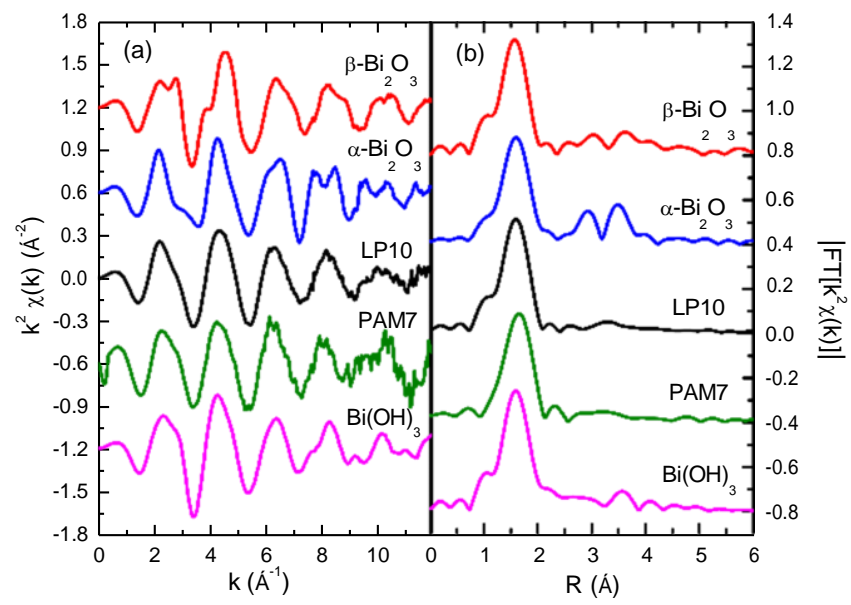

Fig. 6 (left panel) Comparison of the experimental $\mathrm{Bi} \mathrm{L}_{3}$-edge EXAFS spectra of the representative LP10 and PAM7 samples and the reference compounds $\alpha-\mathrm{Bi}_{2} \mathrm{O}_{3}, \beta-\mathrm{Bi}_{2} \mathrm{O}_{3}$ and $\mathrm{Bi}(\mathrm{OH})_{3}$. (right panel) Corresponding FT's. The spectra have been vertically shifted for the sake of clarity.
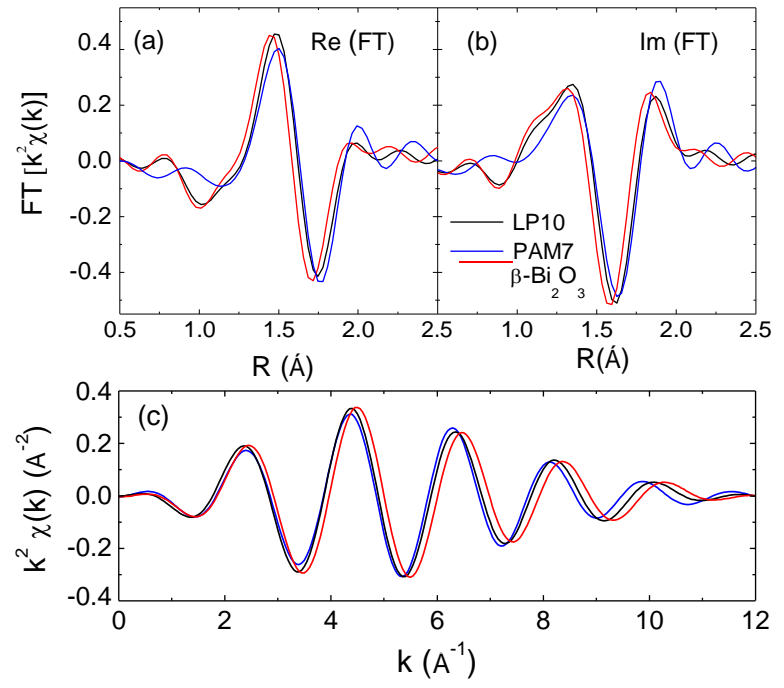

Fig. 7 Detail (0.5 $\AA$ - $2.5 \AA$ range) of the real (a) and imaginary (b) parts of the Fourier transform corresponding to the $\mathrm{Bi}_{3}$-edge EXAFS of the LP10 (black), PAM7 (blue) and $\beta-\mathrm{Bi}_{2} \mathrm{O}_{3}$ (red) samples. (c) Fourier back-transform showing the shift in the contribution corresponding to the first peak.

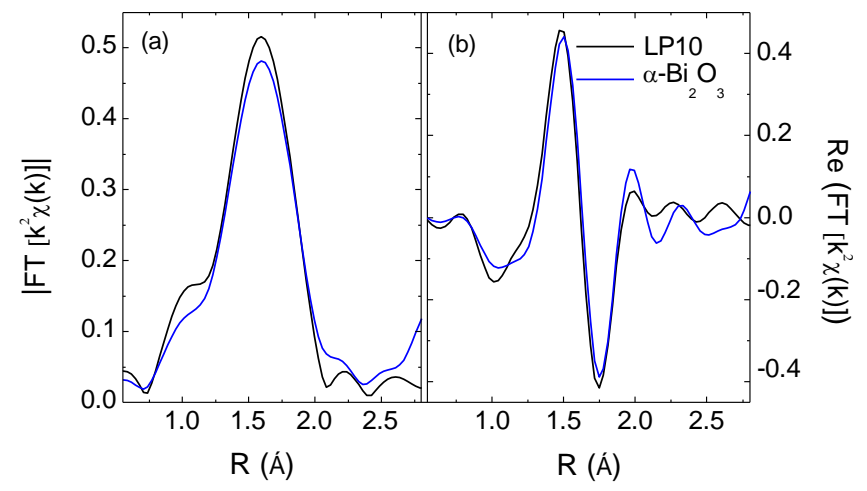

Fig. 8 Comparison of the modulus (a) and real part (b) of the Fourier transform obtained from the $\mathrm{Bi}_{3}$-edge EXAFS spectra of the $\mathrm{LP} 10$ (black) and the $\alpha-\mathrm{Bi}_{2} \mathrm{O}_{3}$ (blue) samples.

$\AA$ ) than in our samples (Fig. 7). As for the $\alpha$ - $\mathrm{Bi}_{2} \mathrm{O}_{3}$ case, although the $\mathrm{Bi}-\mathrm{O}$ distance matches with that of our samples, the profile of the FT corresponding to the first shell is slightly different for $\alpha-\mathrm{Bi}_{2} \mathrm{O}_{3}$ and LP10. And this profile changes from one sample to another. As it can be seen in the real part of the FT (Fig. 8), the region around $\mathrm{R} \sim 1.9-2.4 \AA$ does not match for both samples. Since the profile at the region 1-2.5 $\AA$ is determined by the details of the first coordination shell (number of atoms and distance), it can be concluded that in our samples the oxygen atoms in the first shell around $\mathrm{Bi}$ are arranged in a slightly different way than those in $\alpha-\mathrm{Bi}_{2} \mathrm{O}_{3}$. A more quantitative comparison of the two environments has been included in the supplementary information. Similarly, the comparison to $\mathrm{Bi}(\mathrm{OH})_{3}$ shows that the environments around $\mathrm{Bi}$ are very close for both $\mathrm{Bi}(\mathrm{OH})_{3}$ and LP10. Still, the profile of the first peak of LP10 is slightly different to that of $\mathrm{Bi}(\mathrm{OH})_{3}$.

More importantly, the analysis of the second shell does not show evidence of a $\mathrm{Bi}-\mathrm{Bi}$ (neither of $\mathrm{Bi}-\mathrm{Fe}$ ) scattering path. The almost unnoticeable hump at 232 . 2 does not shoy the
double peak profile one would expect for a Bi scatterer. More-

over, its relative amplitude does not grow with increasing kweight as one can expect for a shell containing atoms with larger atomic number than oxygen. This is displayed in Fig. 9, where the FT spectra are plotted for different k-weights and normalized to the intensity of the first peak. According to this comparison the feature at $\sim 3.2 \AA$ is, most likely, due to scattering from light atoms, such as $\mathrm{O}$ or $\mathrm{H}$. The same conclusion is drawn from a Fourier back-transform analysis of this feature (in the supplementary information).

The results of the combined XANES and EXAFS analysis indicate that, in the case of the samples synthesized by laser pyrolysis or precipitation in aqueous media, the order around $\mathrm{Bi}$ is short-range and extends only to one coordination shell made of oxygen atoms. Moreover, the geometry of this first 


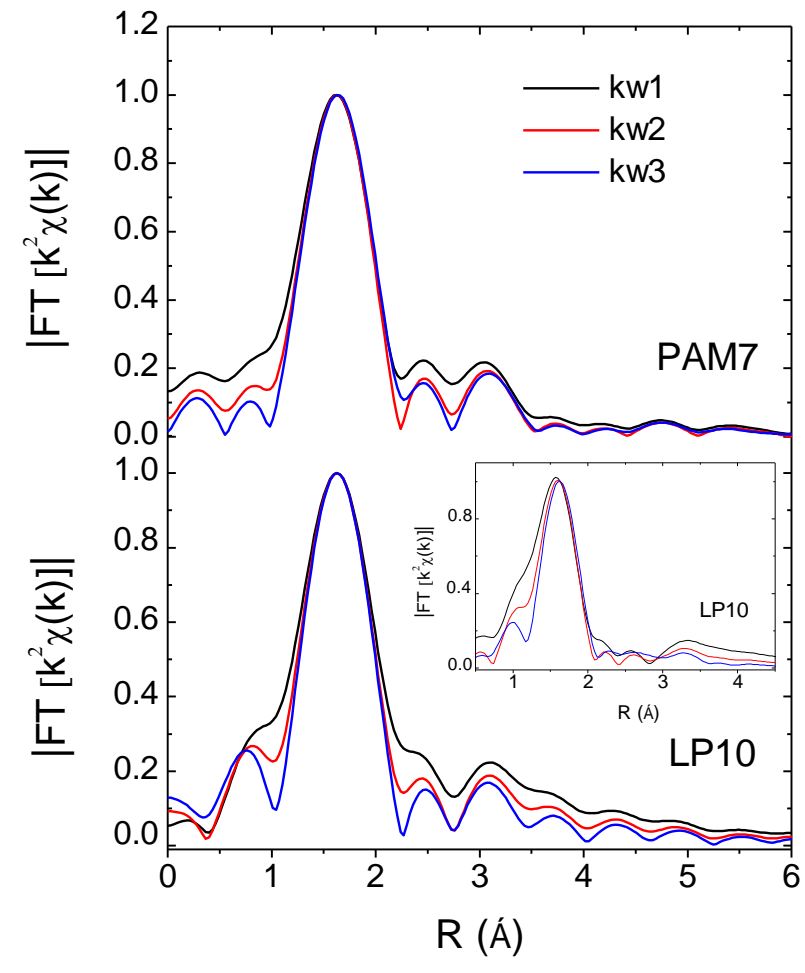

Fig. 9 Bi L3-edge FT (modulus, 2.5-8.5 Åk-range ) plotted for different k-weights and rescaled so that the first shell has the same amplitude with all three k-weights. Inset: same comparison for a k-range from 2.0 to $10.0 \AA$. shell cannot be accurately associated to that of $\alpha-\mathrm{Bi}_{2} \mathrm{O}_{3}$ or $\beta$ $\mathrm{Bi}_{2} \mathrm{O}_{3}$ compounds. As for the analysis of the second shell, the almost unnoticeable hump at $\sim 3.2 \AA$ does not show evidence of a Bi-Bi (neither of Bi-Fe) scattering path. Not having a $\mathrm{Bi}-\mathrm{Bi}$ scattering path, i.e. a $\mathrm{Bi}-\mathrm{O}-\mathrm{Bi}$ coordination, one can hardly say that the $\mathrm{Bi}$ is in $\mathrm{a}_{2} \mathrm{O}_{3}$ crystalline structure, even disordered. Besides, certainly, a disordered $\alpha-\mathrm{Bi}_{2} \mathrm{O}_{3}$ would give rise to a wider and less intense peak in the corresponding Fourier transforms but not to an almost complete extinction of the peak. Therefore, the formation of a defined $\mathrm{Bi}_{2} \mathrm{O}_{3}$ phase is discarded. Similarly, the hypothesis of $\mathrm{Bi}$ embedded in the Fe oxide structure can be undoubtedly discarded since in that case a clear second coordination shell should be seen.

From the above results one cannot rule out the hypothesis of having an entirely amorphous $\mathrm{Bi}$ oxide (i.e. $\mathrm{BiO}_{x}$, where the structure and even the atomic proportion are not defined and no Bi-O-Bi coordination is mandatory at $\sim 3-4 \AA$ ). However, taking into account the close resemblance of the EXAFS signals of LP10 and PAM7 to that of the bismuth hydroxide commercial reference, the k-weight dependence of our FTs, the small $\mathrm{Bi}$ concentration and the presence of hydrogen in the synthesis procedure, these results suggest that $\mathrm{Bi}$ may be in a Bi-hydroxide-like form where the formation of $\mathrm{Bi}-\mathrm{O}-\mathrm{Bi}$ bridges gets prevented by the non-bridging hydroxyl groups rather than in small, amorphous Bi oxide clusters. This would explain that $\mathrm{Bi}_{2} \mathrm{O}_{3}$ seeds are not formed avoiding the formation of a continuous layer and a well defined core-shell interface. Moreover, the presence of $\mathrm{OH}$ groups has been confirmed by TGA measurements (in the supplementary information).

Therefore, our results agree with bismuth being in a distorted octahedral, oxygen-based, local environment forming $\left[\mathrm{BiO}_{6-x}(\mathrm{OH})_{x}\right]$ units (or similar) and being bonded through hydroxyl groups or adsorbed by van der Waals forces to the surface of the nanoparticle. The capping around the spinel nanoparticle consists, therefore, of isolated units bonded through intermolecular forces to the surface of the nanoparticle instead of a well formed $\mathrm{Bi}_{2} \mathrm{O}_{3}$ shell bounded to the core by $\mathrm{Bi}-\mathrm{O}-\mathrm{Fe}$ bridging bonds (intramolecular forces). This has strong implications for the stability and biofunctionality of this hybrid structure, which may depart from that expected for magnetite/bismuth oxide core /shell structures. Thus, the $(\mathrm{OH})$ groups in the $\left[\mathrm{BiO}_{6-x}(\mathrm{OH})_{x}\right]$ unit can interact with other compounds by attaching or exchanging new ligands and giving rise to further surface functionalization.

\section{5 $\mathrm{Bi}_{3}$-edge XANES and EXAFS- TD03 sample}

Regarding the TD03 sample, the profile of the FT of the EXAFS signal shows the presence of a second coordination shell in addition to the first oxygen shell, indicating longer range order around Bi (Fig. 10). For this sample, the amount of 
bismuth is smaller $(0.3 \%)$ than for the other samples. Consequently, the XAS signal is very small and with a poor signalto-noise ratio and so doubts may be cast on any precise conclusion drawn form this spectrum. However, even though an accurate analysis cannot be done for this sample, our data clearly indicate that the thermal decomposition method gives rise to Bi local environments different from those obtained by laser pyrolysis or precipitation in aqueous media.

The analysis of the feature at $\sim 3 \AA$ discards a Bi-Bi origin since it should give rise to a double peak in the FT and to a double hump profile in the filtered EXAFS oscillations. On the other hand, the comparison of the filtered EXAFS corresponding to the second peak with the FEFF simulated $\mathrm{Bi}-\mathrm{O}$ and $\mathrm{Bi}$ Fe EXAFS contributions suggests that it is mainly made of oxygen atoms (or any other light atoms). Nevertheless, an additional contribution from Fe neighbors cannot be discarded. In fact, this FT resembles, to some extent, that found at the Fe K edge (see middle panel Fig. 2 ). The FT corresponding to the Bi $\mathrm{L}_{3}$ edge can be roughly accounted for with a first shell of oxygen atoms at $2.09 \AA$, and a second shell of oxygen and iron atoms at $3.49 \AA$, which corresponds to a $0.2 \AA$ expanded tetrahedral $\mathrm{Fe}$ site in $\mathrm{Fe}_{3} \mathrm{O}_{4}$. The environment around $\mathrm{Bi}$ in $\mathrm{BiFeO}_{3}$ has also been tested but it has been excluded since its second shell is at a much smaller distance. In the case of the samples made by thermal decomposition, the synthesis temperature, $300{ }^{\circ} \mathrm{C}$, is higher than for the other two methods. This could favor that the $\mathrm{Bi}$ atoms bond strongly to the iron oxide. Moreover, the oleic acid among the nanoparticles has a high interaction with these nanoparticles. ${ }^{41}$ This could also help tokeep the Biatoms strongly bonded to the surface of the nanoparticle and in a more ordered environment. In this respect, it cannot be discarded the idea of $\mathrm{Bi}$ atoms being also bonded to the oleic acid, $\left(\mathrm{CH}_{3}\left(\mathrm{CH}_{2}\right)_{7} \mathrm{CH}=\mathrm{CH}\left(\mathrm{CH}_{2}\right)_{7} \mathrm{COOH}\right)$. However, these bonds alone could hardy explain the second peak at the FT since, although a precise value for the number of atoms in the second shell cannot be given at this point, the high amplitude of this peak indicates a high coordination number.

\section{Conclusions}

The structural characterization of Bi-doped iron oxide nanoparticles grown by three different methods has been carried out by X-ray absorption spectroscopy recorded at the $\mathrm{K}$ edge of $\mathrm{Fe}$ and the $\mathrm{L}_{3}$ edge of $\mathrm{Bi}$. Our results discard the formation of $\mathrm{Bi}$ metal (Bi-Bi direct bonds). In addition, in the case of particles grown by laser pyrolysis or precipitation in aqueous media, they show that the $\mathrm{Bi}$ atoms neither enter in the iron oxide spinel lattice nor form any other Bi oxide. The bismuth atoms are in an oxygen-based local environment whose order extends only to one coordination shell. Contrary to expectations, the results discard the possibility of having a well defined core-shell (magnetite-bismuth oxide) structure.

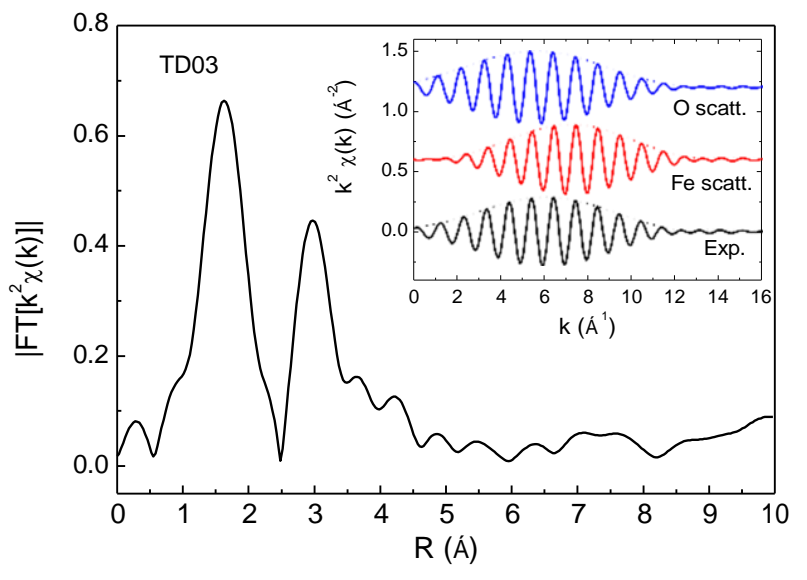

Fig. 10 Modulus of the FT of the experimental Bi $\mathrm{L}_{3}$-edge EXAFS spectra of the TD03 sample (inset) Real part of the second shell Fourier filtered EXAFS oscillaltions.

Our data agree with a hybrid structure made of an iron oxide core and a capping consisting on isolated $\left[\mathrm{BiO}_{6_{-}}(\mathrm{OH})_{x}\right]$ (or similar) units bonded through chemisorption with hydroxyl groups to the surface of the nanoparticle. On the other hand, in the sample grown by thermal decomposition, the longer-range ordered environment suggests that the $\mathrm{Bi}$ atoms are embedded in the spinel lattice, either at the Fe tetrahedral site or at the surface of the nanoparticle but more strongly bonded to the iron oxide. The presented results provide valuable information to better understand the physicochemical properties of Bi-doped magnetite nanoparticles as well as their possibilities for further functionalization and application as double contrast agents for imaging.

\section{Acknowledgements}

This work was partially supported by Spanish MAT201127573-C04-04, MAT-2010-16022, MAT2010-09346-E, MAT2011-23641 and by the Aragón DGA NETOSHIMA grant. The synchrotron radiation experiments were performed at the XAFS beamline of Elettra (Proposal No. 20115379). M. A. Laguna-Marco acknowledges CSIC for a JAE-Doc contract. R. Boada acknowledges support from the Ministerio de Economa y Competitividad of Spain. A. G. R. acknowledges financial support from the Spanish Ministerio de Educacin, through Programa Nacional de Movilidad de Recursos Humanos of Plan Nacional of I-D+i 2008-2011. We thank V. Raghavendra Reddy and C. Meneghini for providing us the reference spectra of $\mathrm{BiFeO}_{3}$. We acknowledge $\mathrm{F}$. Balas and M.P. Morales for useful discussions. 


\section{References}

1 J. Cheon and J.-H. Lee, Accounts of Chemical Research, 2008, 41, 1630.

2 J. H. Lee, J. W. Kim and J. Cheon, Mol Cells, 2013, 35, 274.

3 S. Laurent, D. Forge, M. Port, A. Roch, C. Robic, L. V. Elst and R. N. Muller, Chem. Rev., 2008, 108, 2064.

4 A. G. Roca, R. Costo, A. F. Rebolledo, S. Veintemillas-Verdaguer, P. Tartaj, T. González-Carreño, M. P. Morales and C. J. Serna, J. Phys., D: Appl. Phys., 2009, 42, 224002.

5 P. Tartaj, M. P. Morales, T. González-Carreño, S. Veintemillas-Verdaguer and C. J. Serna, Adv. Mater, 2011, 23, 5243.

6 C. Corot, P. Robert, J.-M. Ide and M. Port, Advanced Drug Delivery reviews, 2006, 58, 1471.

7 J. H. Lee, Y. M. Huh, Y. W. Jun, J. W. Seo, J. T. Jang, H. T. Song, S. Kim, E. J. Cho, H. G. Yoon, J. S. Suh and J. Cheon, Nat. Med., 2007, 13, 95.

8 L. Sun, Y. Zang, M. Sun, H. Wang, X.Zhu, S. Xu, Q. Yang, Y. Liu and Y. S. and, J. Colloid Interface Sci., 2010, 350, 90.

9 N. Lee, S. H. Choi and T. Hyeon, Adv Mater, 2013, 25, 2641.

10 G. G. Briand and N. B. Burford, Chem. Rev., 1999, 99, 2601.

11 W. A. Kalender, Physics in medicine and biology, 2006, 51, R29-43.

12 X. Pan, J. Siewerdsen, P. J. L. Riviere and W. A. Kalender, Medical physics, 2008, 35, 3728.

13 O. Rabin, J. M. Perez, J. Grimm, G. Wojtkiewicz and R. Weissleder, Nat Mater, 2006, 5, 118-122.

14 M. Andrés-Vergés, M. P. Morales, S. Veintemillas-Verdaguer, F. J. Palomares and C. J. Serna, Chem. Mater., 2012, 24, 319.

15 M. Andrés-Vergés, R. Costo, A. G. Roca, J. F. M. G. F. Goya, C. J. Serna and M. P. Morales, J Phys D Appl Phys, 2008, 41, 134003.

16 S. Veintemillas-Verdaguer, M. P. Morales and C. J. Serna, Mater Lett, 1998, 35, 227.

17 M. C. Bautista, O. Bomati-Miguel, M. P. Morales, C. J. Serna and S. Veintemillas-Verdaguer, Journal of Magnetism and Magnetic Materials, 2005, 293, 20.

18 A. G. Roca, S. Veintemillas-Verdaguer, M. Port, C. Robic, C. J. Serna and M. P. Morales, Journal of Physical Chemistry B, 2009, 113, 7033.

19 A. D. Cicco, G. Aquilanti, M. Minicucci, E. Principi, N. Novello, A. Cognigni and L. Olivi, J. Phys.: Conf. Ser., 2009, 190, 012043.

20 D. E. Sayers and B. Bunker, in X-Ray Absorption: Principles, Applications, Techniques of EXAFS, SEXAFS, and XANES, ed. D. C. Koningsberger and R. Prins, Wiley: New York, 1988, ch. 6.

21 M. Newville, J. Synchrotron Rad., 2001, 8, 322.

22 B. Ravel and M. Newville, J. Synchrotron Rad., 2005, 12, 537.

23 C. R. Natoli, D. Misemer, S. Doniach and F. Kutzler, Phys. Rev. A, 1980, 22, 1104.

24 M. Benfatto and S. D. Longa, J. Synchrotron Rad., 2001, 8, 1087.

25 J. Chaboy, H. Maruyama and N. Kawamura, J. Phys.: Condens. Matter, 2007, 19, 216214

26 J. Chaboy, N. Nakajima and Y. Tezuka, J. Phys.: Condens. Matter, 2007, 19, 266206.

27 C. Guglieri and J. Chaboy, J. Phys. Chem. C, 2010, 114, 19629.

28 C. Guglieri, E. Céspedes, C. Prieto and J. Chaboy, J. Phys.: Condens. Matter, 2011, 23, 206006

29 S. Díaz-Moreno, A. Muñoz-Páez and J. Chaboy, J. Phys. Chem. A, 2000, 104, 1278.

30 J. Chaboy and S. Quartieri, Phys. Rev. B, 1995, 52, 6349.

31 J. Chaboy, J. Synchr. Rad., 2009, 16, 533.

32 J. Chaboy, A. Muñoz-Páez, F. Carrera, P. Merkling and E. SánchezMarcos, Phys. Rev. B, 2005, 71, 134208.

33 M. O. Krause and J. Oliver, J. Phys. Chem. Ref. Data, 1979, 8, 329.

34 C. Piquer, M. A. Laguna-Marco, A. G. Roca, R. Boada, C. Guglieri and J. Chaboy, J. Phys. Chem. C, 2014, 118, 1332-1346.

35 Introduction to XAFS: A Practical Guide to X-ray Absorption Fine Struc- ture Spectroscopy, ed. G. Bunker, Cambridge University Press, U.K., 2010.

36 P. Glatzel, G. Smolentsev and G. Bunker, J. Phys.: Conf. Ser., 2009, 190, 012046.

37 F. Farges, Phys. Chem. Miner, 2009, 36, 463.

38 A. Zitolo, V. Migliorati, G. Aquilanti and P. D'Angelo, Chem. Phys. Lett., 2014, 591, $32-36$.

39 J. Chaboy and S. Díaz-Moreno, J. Phys. Chem. A, 2011, 115, 2345-2349.

40 H. A. Harwig, Z. Anorg. Allgem. Chem., 1978, 444, 151.

41 A. G. Roca, M. P. Morales, K. O'Grady and C. J. Serna, Nanotechnology, 2006, 17, 2783. 